\title{
Coherence, Muller's Ratchet, and the Maintenance of Culture
}

\author{
(Accepted for publication: A shortened version will \\ appear in Philosophy of Science 82(5), 2015.) \\ Marshall Abrams (mabrams@uab.edu) \\ Department of Philosophy \\ University of Alabama at Birmingham
}

November 9, 2014

\begin{abstract}
I investigate the structure of an argument that culture cannot be maintained in a population if each individual acquires a given cultural variant from a single person. I note two puzzling consequences of the argument: It appears to conflict with (a) many models of cultural transmission and (b) real-world cases of cultural transmission. I resolve the first puzzle by showing that one of the models central to the argument is conceptually analogous and mathematically equivalent to one used to investigate the evolution of sexual reproduction. This analogy clarifies what assumptions are crucial to the argument concerning cultural transmission. I resolve the second puzzle by arguing that probabilistic models of epistemological coherence can be reinterpreted as models of mutual support between cultural variants. I develop a model of cultural transmission illustrating this proposal. I suggest that real-world cases that seem to conflict with the original argument may in fact be instances in which mutually supporting cultural variants are learned from different individuals.
\end{abstract}

\section{Introduction}

I investigate the structure of an argument that culture cannot be maintained in a population if each individual acquires a given cultural variant from a single person. I note two puzzling consequences of the argument: It appears to conflict with (a) many models of cultural transmission and (b) real-world cases of cultural transmission. I resolve the first puzzle by showing that one of the models central to the 
argument is conceptually analogous and mathematically equivalent to one used to investigate the evolution of sexual reproduction. This analogy clarifies what assumptions are crucial to the argument concerning cultural transmission. I resolve the second puzzle by arguing that probabilistic models of epistemological coherence can be reinterpreted as models of mutual support between cultural variants. I develop a model of cultural transmission illustrating this proposal. I suggest that real-world cases that seem to conflict with the original argument may in fact be instances in which mutually supporting cultural variants are learned from different individuals.

Section 2 describes arguments based on models of cultural transmission in (Enquist et al., 2010), and section 3 describes two implications of Enquist et al.'s results that may seem puzzling. Section 4 explains why the evolution of sexual reproduction is an interesting problem in evolutionary biology, and describes Muller's ratchet, a kind of model that motivates one of the proposed evolutionary advantages of sexual reproduction. This is the model that bears mathematical and conceptual parallels to one of Enquist et al.'s models. Section 5 then reinterprets ideas from probabilistic models of coherence in epistemology as ideas about cognitive transitions from one cultural variant to another.

I'll assume that there are at least minor differences in beliefs, knowledge, behavior, attitudes, inclinations, etc. between people within a society, and that such characteristics - "cultural variants" - in one person sometimes promote similar variants in another. ${ }^{1}$ Such processes are called "cultural transmission" or "social learning". "Individual learning", by contrast, occurs when a person learns something on their own, whether through observation of the environment, trial and error, reasoning, or some combination.

\section{One Cultural Parent Makes No Culture}

In this section I summarize arguments made by Enquist, Strimling, Eriksson, Laland, Sjostrand (2010) (henceforth "ESELS"). These authors argue that if a particular cultural variant is always acquired from a single individual - a single "cultural parent" - then it's very difficult for the cultural variant to be maintained in a population. Culture would usually disappear. Thus the maintenance of culture in human populations seems to depend on the fact that we routinely learn from multiple individuals.

\footnotetext{
${ }^{1}$ This concept of a cultural variant does not carry the same connotations as the term "meme", which tends to suggest discreteness, near-perfect replicability, or goal-directedness (cf. Richerson and Boyd 2005; Godfrey-Smith 2009).
} 


\section{Single cultural parents}

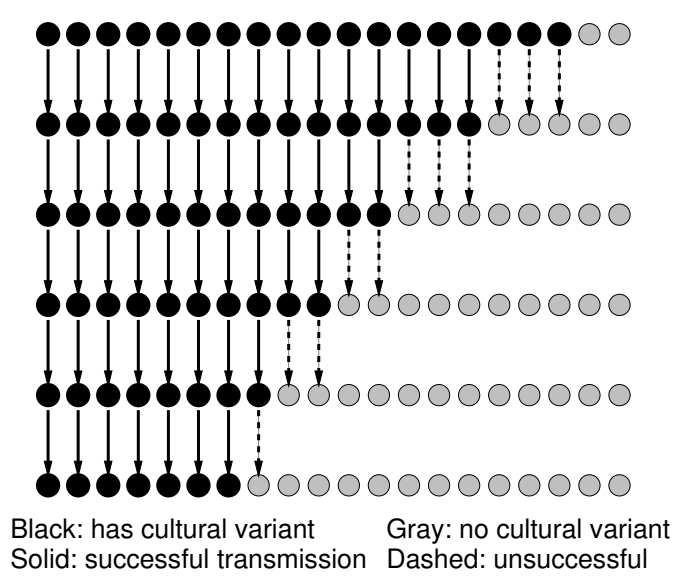

Figure 1: Cultural transmission from randomly chosen "parents" in discrete generations, with time moving from top to bottom. A cultural variant can only be acquired from those who have it (black circles), but transmission (arrows) sometimes fails (dashed arrows).

Suppose that a cultural variant $C$ is either present or not, and assume that cultural transmission is imperfect: When individual $a$ learns from individual $b$, there is a chance that $a$ will fail to learn. Suppose then that each individual learns with probability $p$, from a randomly chosen member of a population in which those with $C$ have relative frequency $x_{t}$ at time $t$. We'll assume that learning occurs in discrete timesteps, or cultural "generations".

ESELS note that the frequency of those with $C$ will be $p^{t} x_{0}$, which decreases to zero unless transmission is perfect. ${ }^{2}$ Figure 1 illustrates this process. In each generation, a fraction $p$ of those with the cultural variant $C$ succeed in transmitting it, so the frequency of $C$ decreases over time. ESELS consider several variations on this model, including (1) models in which multiple learning trials from the same cultural parent are allowed, (2) models in which cultural parents can be chosen because they appear to be successful ("biased transmission"), (3) models in which social learning is combined with individual learning, and (4) models in which possession of a cultural variant confers a fitness advantage that makes the bearer more likely to be available to be chosen as a cultural parent. I won't review all of these models in detail. I note that ESELS showed that that option (1) merely slows down the eventual loss of culture. Option (2) can maintain culture, but only under some parameter values. With option (3), it turns out that under plausible parameter val-

\footnotetext{
${ }^{2}$ I ignore possible effects of random drift in cultural transmission processes throughout this paper.
} 
ues, culture can only be maintained if individual learning alone could have maintained it. I turn now to option (4).

Suppose that having the cultural variant $C$ provides a fitness benefit. For example, perhaps it makes survival more likely, thus allowing an individual more time to influence others. Let $\alpha \geq 1$ be the ratio between the fitness of those with the cultural variant and the fitness of those without it. Under these assumptions, ESELS argue that if the frequency in the current generation is $x$, the average frequency $x^{\prime}$ in the next generation is:

$$
x^{\prime}=\frac{p \alpha x}{p \alpha x+(1-p x)} .
$$

Here $p \alpha x$ is the frequency of successful cultural transmission: $\alpha x$ represents frequency of $C$ among chosen cultural parents, and $p$ is the probability of successful transmission of $C .(1-p x)$ is the frequency of transmissions that don't occur, either because the (randomly) chosen parent lacks $C$, or because the cultural transmission nevertheless failed.

The frequency of $C$ in the population will keep changing until an equilibrium is reached in which $x^{\prime}=x$. ESELS show that this equilibrium $\hat{x}$ is:

$$
\hat{x}=\frac{\alpha p-1}{p(\alpha-1)}
$$

ESELS argue that the equilibrium is nonzero only for parameter values that are rare in nature. For example, if the probability $p$ of successful transmission $0.9-\mathrm{a}$ very high value - the ratio between fitnesses of those with and without $C$ must be greater than about 1.112 in order for culture to be maintained. This is an unusually large fitness advantage.

On the other hand, ESELS show that allowing individuals to learn from two or more cultural parents can easily maintain culture, even without a fitness advantage. The reason is that even if a chosen cultural parent lacks the cultural variant $C$, or fails to transmit it, there is the the possibility that another randomly chosen cultural parent will successfully transmit $C$ (figure 2). Having a "backup" teacher allows learners to recover, often, from a failure of cultural transmission. In this model, for $n$ randomly chosen cultural parents, the frequency $x^{\prime}$ in the next generation is: ${ }^{3}$

$$
x^{\prime}=1-(1-p x)^{n}
$$

\footnotetext{
${ }^{3}$ The frequency of successful transmissions is equal to the probability $\mathrm{P}\left(T_{1} \vee \cdots \vee T_{n}\right)$ of successful transmission $T_{i}$ from any of the $n$ cultural parents. Since successful transmission from more than one parent is not ruled out, the probability of at least one successful transmission is equal to $\sum_{i=1}^{n} \mathrm{P}\left(T_{i}\right)$ minus a complex function of probabilities of conjunctions of the $T_{i}$ 's. A routine technique is to simplify such a calculation using De Morgan's law, transforming the above disjunction into $\mathrm{P}\left(\neg\left(\neg T_{1} \& \cdots \& \neg T_{n}\right)\right)$. The frequency is then equal to (3), since the events $T_{i}$ are independent.
} 


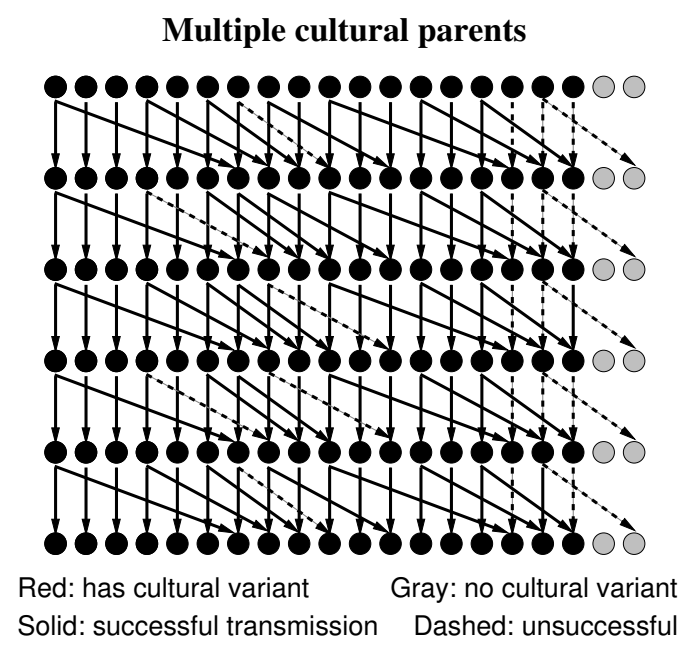

Figure 2: Each diagonal arrow represents cultural transmission from a second cultural parent. See caption for figure 1 for the meaning of other components.

ESELS show that there is a nonzero equilibrium if and only if $p n>1$. On this model, culture will be maintained as long as the probability of transmission is not too low, and the number of cultural parents is high enough. For example, if each learner has two cultural parents, the probability of successful transmission need only be slightly greater than $1 / 2$. Note that this model implies, in effect, that maintenance of culture requires robustness resulting from multiple processes with at least poorly correlated errors (Wimsatt, 2007).

\section{Two puzzles}

There are two implications of ESELS' argument that may initially seem puzzling. First, many mathematical models of cultural change seem to allow single-parent culture to be maintained. What is the crucial difference in ESELS' model that prevents this? Consider, for example Rogers' (1988) model, which concerns two behaviors, each of which is better adapted to a different environment. Some individual chose a behavior based on individual learning. Others simply copy the behavior of a randomly chosen individual. Rogers shows that at equilibrium, the fitness of social learners is equal to that of individual learners. If we think of the lack of cultural variant $C$ in ESELS' models as a a kind of null cultural variant (which ESELS call $N$ ), the loss of $C$ from a population corresponds to fixation of its absence, i.e. fixation of $N$. However, in Rogers' models, neither of the two cultural variants represented goes to fixation, under a wide range of parameter values. Rogers' model and ESELS' models clearly depend on different assumptions. What are the 

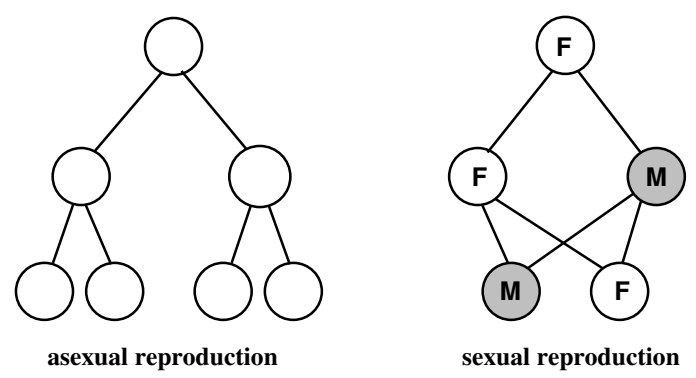

Figure 3: Biological descendant relations in a species limited to two offspring per egg-producing organism.

assumptions in ESELS' model that allow culture to disappear? A closely related question is this: Why not simply switch these labels in ESELS' model? Then it would be inevitable that culture would spread with single-parent transmission! I address these questions in in section 4 .

The second implication of ESELS' argument is that it seems to conflict with realworld cases of single-parent transmission. Is it really true, empirically, that culture is rarely maintained through single-parent transmission? To take an example from the cultures of modern industrialized societies, from how many people did you learn long division? How many people taught your teacher? Granted, this isn't much of an argument: Rather than collecting real data, I'm appealing to anecdotal evidence. Nevertheless, there is some reason to wonder whether, or how, the real world might conflict with ESELS' conclusions. I address these questions in section 5 .

\section{Muller's ratchet}

In this section I describe a widely-investigated question concerning the evolution of sexual reproduction. I then describe Muller's ratchet, a class of models that are central to one of several proposed answers to the question. I draw inferences concerning ESELS argument concerning single-parent culture from the close conceptual and mathematical parallels between a recent formulation of Muller's ratchet and one of ESELS' models described above.

Sexually reproducing species evolved from asexual species, and in some species, both kinds of reproduction occur. Even in the case of organisms that simply leave their eggs behind, producing eggs requires significant energy and material resources, which limits the number of offspring that females or asexual organisms can produce. An argument due to Maynard Smith (1978) showed that asexual organisms can have twice as many grand-offspring as sexually reproducing females, with no 
additional energy expenditure. This is illustrated in figure 3. Suppose, for example, that each egg-producing organism can produce two offspring in a population of asexual reproducers. Each organism will then produce four grand-offspring. If one organism has a new mutation that causes offspring to reproduce sexually, and produces one male and one female, they must mate to produce offspring, producing only two offspring between them. This argument can be generalized (Maynard Smith, 1978). The upshot is that since sexual reproduction reduces the number of descendants of an organism by half, it must generate an enormous fitness benefit in order for it to have been selected for. There are a number of proposed explanations of the benefit of sexual reproduction that are under active investigation (Otto and Lenormand, 2002). I focus only on Muller's ratchet, a class of models that show that in the absence of sexual reproduction and recombination, deleterious (disadvantageous) mutations will accumulate in a population and eventually cause its extinction.

Muller's ratchet is based on the biologically plausible assumptions that beneficial mutations are rare, that strongly deleterious mutations will quickly be selected out of a population, and that backmutations that undo a mildly deleterious mutation are improbable. Over time, lineages accumulate different numbers of mildly deleterious mutations. Since the evolutionary costs of these mutations are small, lineages with the fewest deleterious mutations will occasionally be lost due to random genetic drift. At that point, all members of the population have more deleterious mutations than those fitter members that were lost, so there is almost no possibility of creating descendants with fewer deleterious mutations. We say that Muller's ratchet has clicked. This process gradually leads to the accumulation of deleterious mutations, and ultimately, to the extinction of the population. Sexual reproduction with recombination provides an escape from the ratchet, though. Recombination combines different segments of two individuals' chromosomes in order to produce an offspring's chromosomes. This allows some offspring to have fewer deleterious mutations than either of their parents, thus preventing the inevitable decline of fitness in the population as a whole that would result from Muller's ratchet.

Most Muller's ratchet models (e.g. Haigh 1978) divide the population into classes of organisms, each with a different number of deleterious mutations. Waxman and Loewe's (2010) "truncated Ratchet" model instead has only two classes: The class of individuals with the fewest deleterious mutations, and a class containing all other individuals. A click of the ratchet is the loss of the fittest class, and a subset of the other class then becomes the new fittest class. Let $\mu$ be the per-organism probability of a deleterious mutation; $1-\mu$ is then the probability that an individual 
will remain in the fittest class. Let $\sigma$ be the average fitness cost from deleterious mutations due to being in the less fit class of organisms, and $1-\sigma$ the fitness of the members of the fitter class. The probability that a deleterious mutation will be undone by a backmutation is so small that it's reasonable to treat it as zero, and we ignore beneficial mutations as well.

Waxman and Loewe then derive the following frequency $x^{\prime}$ of the fittest class in the next generation, starting from the frequency $x$ in the current generation:

$$
x^{\prime}=\frac{(1-\mu) x}{(1-\sigma)+\sigma(1-\mu) x}
$$

(It's not completely straightforward to interpret the components of this formula.) By equating $x$ and $x^{\prime}$, Waxman and Loewe derive the equilibrium frequency $\hat{x}$ of the fittest class:

$$
\hat{x}=\frac{\sigma-\mu}{\sigma(1-\mu)}
$$

I want to compare ESELS' model with selection and Waxman and Loewe's model. Both sets of models have a parameter representing the probability of error-free transmission. In ESELS' model, this is $p$, the probability of transmitting the cultural variant $C$ without error. In Waxman and Loewe, $1-\mu$ is the probability of reproduction without a deleterious mutation. Both sets of models also have a parameter representing the fitness difference between two states. ESELS $\alpha$ is the ratio between the fitnesses of a cultural variant $C$ and its absence, $N$. Waxman and Loewe instead represent the relationship between fitnesses of the fittest and less fit classes with a difference parameter $\sigma$. We can equate the two sets of parameters, with ESELS' parameters on the left, and Waxman and Loewe's on the right:

$$
\begin{aligned}
& \text { ESELS } \text { Waxman and Loewe } \\
& p=1-\mu \\
& \alpha \times(\text { fitness of } N)=1 \\
&(\text { fitness of } N)=1-\sigma
\end{aligned}
$$

When we equate the parameters, it turns out that Waxman and Loewe's truncated ratchet is mathematically equivalent to ESELS' single-parent model with fitness: Equation (1) is equivalent to equation (4), and equation (2) is equivalent to equation (5). This close relationship between ESELS' model and Waxman and Loewe's truncated ratchet shows that failing to learn from a single cultural parent is so closely analogous to acquiring a deleterious mutation in an asexual species, that both relationships can be modeled in the same way. Similarly, in either model, the loss of beneficial characteristics can be avoided by allowing information to be transmitted from (at least) two parents. 
Importantly, the emphasis in Muller's ratchet models on relations between certain probabilities highlights similar relations in the ESELS models. As in other Muller's ratchet models, Waxman and Loewe's model makes the probability of a deleterious mutation (small, but positive) and no mutation (large) significantly different. Importantly, Muller's ratchet models set the probability of undoing a deleterious mutation equal to zero. Analogously, in ESELS' single-parent model with with fitness, transmission of a beneficial trait $C$ has a significant probability of failure, and there is no chance of undoing the loss of $C$ from a lineage. This is what creates an inevitable loss of culture, just as Muller's ratchet creates an inevitable loss of fitter variants. By comparison, Rogers' (1988) model gives equal probability to learning either of two cultural variants. Models of that kind are appropriate for cultural variants that can easily replace each other in a given cultural context. ESELS' models, on the other hand, seem most appropriate for cultural variants that are difficult to learn in the first place, and that are readily lost without any replacement.

\section{Coherence}

I noted above that it seems somewhat plausible that there are cases of single-parent transmission. In this section I suggest that probabilistic coherence measures inspired by C.I. Lewis's (1946) concept of congruence can, with a slight reinterpretation, be used to understand how a kind of single-parent cultural transmission - or at least the illusion of single-parent cultural transmission-can maintain culture indefinitely.

C.I. Lewis defined his coherence measure, known as congruence, as follows:

A set of statements, or a set of supposed facts asserted, will be said to be congruent if and only if they are so related that the antecedent probability of any one of them will be increased if the remainder of the set can be assumed as given premises. (Lewis, 1946, p. 338)

Two propositions $C_{1}$ and $C_{2}$ are thus congruent iff:

$$
\begin{aligned}
& \mathrm{P}\left(C_{1} \mid C_{2}\right)>\mathrm{P}\left(C_{1}\right) \\
& \mathrm{P}\left(C_{2} \mid C_{1}\right)>\mathrm{P}\left(C_{2}\right) .
\end{aligned}
$$

For two propositions, this relationship can also be captured by requiring that Shogenji's (1999) coherence measure

$$
\frac{\mathrm{P}\left(C_{1} \& C_{2}\right)}{\mathrm{P}\left(C_{1}\right) \mathrm{P}\left(C_{2}\right)}
$$


be greater than 1 .

Lewis's congruence does not fit all intuitions about the role of coherence in justification (Olsson, 2005). Olsson (2005) has shown, for coherence measures such as Shogenji's, that greater coherence does not consistently imply a higher probability of truth of the propositions considered. However, my concern below will not be with truth-conduciveness. I'll treat the probabilities above as probabilities of believing one proposition given believing the other, or more generally, as probabilities of acquiring one cultural variant given the other. This is in effect translates a justificatory relation into a cognitive or behavioral relation.

In real human cultural transmission, we don't only learn isolated bits of information. Research suggests that we remember and can use what we learn more effectively if it is systematically related to other things we learn (Bransford and National Research Council, 2000). Roughly, it helps if different things we learn are related and mutually supporting. ${ }^{4} I^{\prime} l 1$ suggest that we can capture some aspects of this property of human learning with Lewis's congruence notion, and with various extensions of it. In what follows, I'll focus on the simple case of two cultural variants $C_{1}$ and $C_{2}$ that influence each others' adoption.

First note that ESELS's arguments can be applied simultaneously to two cultural variants $C_{1}$ and $C_{2}$ : If either cultural variant is transmitted only by single cultural parents, it will eventually be lost from the population. This is true whether the two variants are both learned from the same parent, or from distinct randomly chosen parents. Research on learning mentioned above, however, raises the possibility that some cultural transmission of coherent beliefs might help prevent the loss of culture. If $C_{1}$ and $C_{2}$ are congruent, so that each raises the probability of believing the other, could this prevent the loss of culture? It appears that it can only slow down the loss of culture, if both beliefs must be acquired from the same cultural parent. I'll explain why.

Suppose that a learner can acquire all or some of the cultural variants possessed by a randomly chosen cultural parent, where the probability of acquiring any one of the cultural variants is the same, $p$. Suppose also that it's possible for an individual to infer (etc. - see below) a missing cultural variant $C_{i}$ if one has the other cultural variant. We can capture the probability of such an inference by:

$$
\mathrm{P}\left(C_{1} \mid C_{2}\right)=\mathrm{P}\left(C_{2} \mid C_{1}\right)=r>0 .
$$

This is like congruence, or Shogenji's coherence being greater than 1 , since we're assuming that the probability of spontaneously acquiring $C_{1}$, i.e. $\mathrm{P}\left(C_{1} \mid \neg C_{2}\right)$, is zero

\footnotetext{
${ }^{4}$ Proviso: The research summarized in (Bransford and National Research Council, 2000) seems to focus only on formal schooling in industrialized societies. Henrich et al. (2010) argue that many experimental results from industrialized populations do not generalize to all humans.
} 


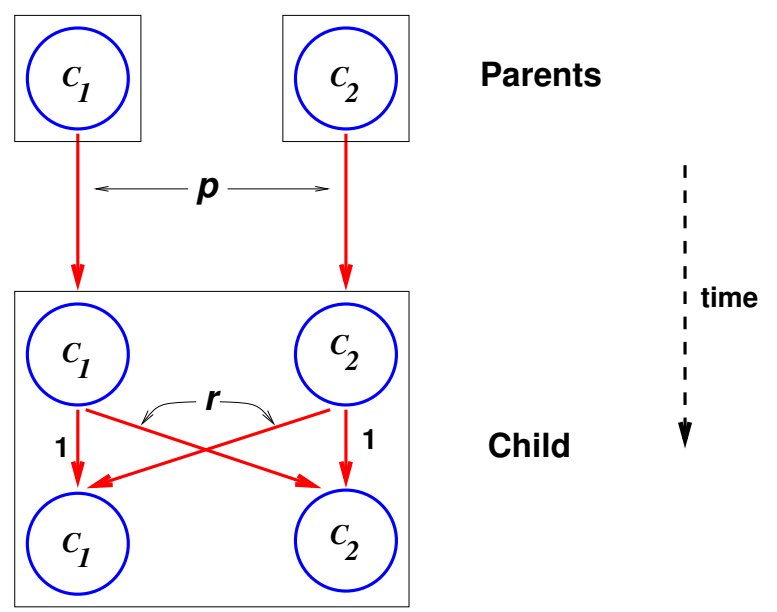

Figure 4: Causal relationships in the cultural variant model described in the text. Rectangles represent persons. Circles represent instances of cultural variant $C_{i}$ held by a person at a time. Labels represent probabilities of succesfully causing (via that path) an instance of a cultural variant to be held, conditional on the variant being held at the earlier time. $p=$ probability of learning from a cultural parent, while $r=$ the probability of "inferring" one cultural variant from the other.

(note $\mathrm{P}\left(C_{1} \mid C_{2}\right)>P\left(C_{1}\right)$ iff $\left.\mathrm{P}\left(C_{1} \mid C_{2}\right)>P\left(C_{1} \mid \neg C_{2}\right)\right)$. ${ }^{5}$. These conditional probabilities in effect link $C_{1}$ and $C_{2}$, so that if a person fails to acquire one of them, but acquires the other, she'll be able to acquire the first anyway, with probability $r$. We can call $r$ a "generalized inference probability", since if $C_{1}$ and $C_{2}$ are beliefs that would cause each other to be held due to rational inference, $r$ is the probability of inferring one from the other, even though there may be other reasons that adopting one cultural variant would cause the other to be adopted.

[More precisely, let $X$ be binary variable with value 1 when the parent has $C_{1}$ and 0 when the parent doesn't, and let $X^{\prime}=1$ represent the learner having $C_{1}$, with $X^{\prime}=0$ representing that it doesn't. Similarly, let $Y$ and $Y^{\prime}$ represent the (other) parent having $C_{2}$ and the learner having $Y^{\prime}$, respectively. Then the claim is that $r=\mathrm{P}_{\neg X \& \neg Y}\left(X^{\prime} \mid \mathrm{do}\left(Y^{\prime}=1\right)\right)>\mathrm{P}_{\neg X \& \neg Y}\left(X^{\prime} \mid \mathrm{do}\left(Y^{\prime}=0\right)\right)=0$, where $\mathrm{P}_{\neg X \& \neg Y}(\alpha)=$ $\mathrm{P}(\alpha \mid \neg X \& \neg Y)$, and do is the operator, defined by Pearl (2009), that sets the value of a variable regardless of what values its causal parents have. A similar claim can be made about $Y^{\prime}$ conditional on do $\left(X^{\prime}=1\right)$, etc. This way of capturing Shogenji coherence requires a cyclic causal dependency that can be be removed by providing additional, within-generation time-indexing of $X^{\prime}$ and $Y^{\prime}$ to the model (figure 4). This suggests that making coherence measures sensitive to cognitive (or other perindividual) causal relations requires extending coherence measures.] 
Let's focus on the best case for using such internal inference processes to maintain culture: Let $r$ be near 1 . Then most individuals with either cultural variant will have the other. Those who have only one of $C_{1}$ and $C_{2}$ will nevertheless be able to produce cultural offspring who have both cultural variants. However, with large $r, C_{1}$ and $C_{2}$ are functioning almost as a unit. The process is roughly the same as what would happen in ESELS' single-parent model if we increased the transmission probability $p$ : It would take longer for culture to disappear, but it would still do so, eventually.

Suppose, however, that equation (6) holds, but that the cultural parent for each of the two cultural variants is chosen independently for each cultural "child". Different cultural variants are acquired from different cultural parents. Thus the probability of acquiring one cultural variant (e.g. $\left.C_{1}\right)$ given the other $\left(C_{2}\right)$ is $r$. And assume that the parent-to-child transmission probability for each variant is $p$. Then the probability of acquiring $C_{1}$ is similar to probability of acquiring $C$ from either of two cultural parents in equation (3). Let $x$ be the relative frequency of $C_{1}$ in the population, and $y$ the relative frequency of $C_{2}$. Then the frequencies $x^{\prime}$ of $C_{1}$ and $y^{\prime}$ of $C_{2}$ in the next generation are:

$$
\begin{aligned}
& x^{\prime}=1-(1-p x)(1-r p y)=p x+r p y-r p^{2} x y \\
& y^{\prime}=1-(1-p y)(1-r p x)=p y+r p x-r p^{2} x y
\end{aligned}
$$

The first equation, for example, is based on the following reasoning (cf. note 3 ). One can acquire $C_{1}$ directly from the cultural parent chosen to transmit $C_{1}$, with probability $p x$, or fail to do so with probability $1-p x$. Similarly, one can acquire $C_{1}$ with probability $r$ from $C_{2}$ if $C_{2}$ was acquired - which happens with probability $p y$. So failing to succeed by this path is $1-r p x$. The probability of successful transmission of $C_{1}$ is then is the probability of failing to fail to acquire $C_{1}$ by one of the two probabilistically independent paths.

The dynamics of this model are not identical to those of the simple two-parent transmission model characterized by equation (3). However, we can simplify the new model, because iterating equations (7) and (8) quickly causes $C_{1}$ and $C_{2}$ to have the same frequency. To see this note that

$$
\left|x^{\prime}-y^{\prime}\right|=|p x+r p y-p y-r p x|=p(1-r)|x-y| .
$$

But $p(1-r)$ must lie between 0 and 1 , so the difference between the frequency $x$ of $C_{1}$ and the frequency $y$ of $C_{2}$ shrinks in every generation. ${ }^{6}$ Thus for the long term effects of cultural transmission of $C_{1}$ and $C_{2}$ when the inference probability

\footnotetext{
${ }^{6}$ In simulations with a variety of values of $p$ and $r, x$ and $y$ come together within 10 or 20 generations, even with initial values $x=0.01$ and $y=0.99$.
} 
$r$ is the same in both directions, we can ignore the difference between their initial frequencies, and model the change in either frequency as:

$$
x^{\prime}=1-(1-p x)(1-r p x)
$$

When $r$ is high, this equation shares with (3) the property that when one fails to learn a cultural variant from a single chosen cultural parent, another parent is relatively likely to transmit that variant. In this case, however, the transmission from the second parent is indirect, via the other cultural variant $C_{2}$ (cf. figure 2).

The frequency of $C_{1}\left(\right.$ or $\left.C_{2}\right)$ is at equilibrium when $x^{\prime}-x=0$, i.e. when

$$
0=\left(p x+r p x-r p^{2} x^{2}\right)-x=x\left([r p+p-1]-r p^{2} x\right)
$$

The right hand side is equal to zero either when $x=0,{ }^{7}$ or when $(r p+p-1)-r p^{2} x=$ 0 , i.e. when $x$ has the value:

$$
\hat{x}=\frac{r p+p-1}{r p^{2}} .
$$

For example, this equilibrium frequency is approximately 0.9 when $p=r=0.83$. This equation also shows that the equilibrium is greater than zero iff $r p+p>1$, i.e. iff

$$
r>\frac{1-p}{p} \text {. }
$$

Thus there is a nonzero equilibrium whenever the internal inference probability is greater than the ratio between the probabilities of direct transmission failure and success, which holds whenever $p$ and $r$ are not too small. When $p$ and $r$ are equal, they must be greater than about 0.62 for the cultural variants to stabilize at a nonzero value. ${ }^{8}$

I suggest, then, the fact that some cultural variants - perhaps long division - seem to be maintained by transmission from single cultural parents, may be due to the fact that these variants are supported by transmission of other variants from other cultural parents. Long division, for example, is not learned in a vacuum. A variety of closely related mathematical concepts are usually learned first, and subsequent use in other contexts provides additional support for it. Thus it may be that students are able to infer, or at least be reminded of, missing steps in long division when forgotten because of what they learned from multiple cultural parents.

\footnotetext{
${ }^{7}$ When $x=0$, equation (9) is misleading. If (7) and (8) are allowed to iterate, it's possible for one cultural variant, say $C_{2}$, to begin with frequency $y=0$ and still reach a non-zero equilibrium.

${ }^{8}$ In the appendix, I show that when $C_{1}$ and $C_{2}$ have different inference and direct transmission probabilities, a nonzero equilibrium exists iff $r s>(1-p)(1-q) / p q$, where $r$ and $s$ are inference probabilities and $p$ and $q$ are corresponding transmission probabilities.
} 
The preceding model considered only two related cultural variants, but real learning and real culture surely involve more complex relations of support between variants learned. Lewis's (1946) concept of congruence, perhaps formalized as Angere's (2008) $C_{E}$, allows for probabilistic support involving more propositions. Shogenji's (1999) measure of coherence does as well, but in a different way. Schupbach (2011) extends Shogenji's measure to make it sensitive to additional relations of probabilistic support. Fitelson's $(2003 ; 2004)$ coherence measure reflects more relations of probabilistic support between conjunctions of propositions in a given set. It may be that one of these coherence measures, or a related one, though not designed to capture the degree to which a set of cultural variants allow restoration of one from others, will be useful for characterizing such a property.

Although classic treatments of the role of coherence in epistemology discuss it in probabilistic terms (Lewis, 1946; BonJour, 1985), the paradigmatic relations underlying the probabilities were usually thought to be, or to be similar to, logical or explanatory relations (cf. also Lehrer 2000). This makes sense given that coherence was intended to provide justification for beliefs. In giving ideas from epistemological models of coherence in a role in cultural transmission, we have to allow a broader basis for the relevant probabilities, though. When a person comes to adopt cultural variant $C_{1}$ because they have previously adopted variant $C_{2}$, this could be because both cultural variants are beliefs, and they have noticed that $C_{2}$ helps to justify $C_{1}$. This justificatory relationship might be mediated by a great deal of cultural background belief, however. However, other sorts of relationships between beliefs may provide the basis for the probabilistic relationship between acquisition of cultural variants. It may be that given the cultural background that the person has already adopted, there is some nonlogical resonance felt between $C_{1}$ and $C_{2}$.

\section{Conclusion}

Enquist et al. (2010) argued that culture will usually disappear unless individuals learn from multiple "cultural parents". The conceptual and mathematical analogy of one of ESELS' models to Waxman and Loewe's truncated Muller's ratchet model clarified that ESELS' models make the assumptions, unusual among cultural transmission models, that transmission probabilities very assymetric, and that once a person loses a cultural variant, it can never come back among cultural "descendants". This crucial assumption makes ESELS' models relevant to cultural variants that are difficult to learn socially - so that they may not be learned at all-and difficult to learn individually. Modeling relations between cultural variants using ideas from probabilistic measures of coherence in epistemology provides a way of 
modeling the influence of one cultural variant on another. This allows the learning of $C_{2}$ from single parents to help maintain $C_{1}$ in the population, even if $C_{1}$ is itself only learned from single parents. Coherence in this sense captures what might be called "inferential robustness": the ability to infer or otherwise learn cultural variants through multiple, redundant paths (Wimsatt, 2007). This way of thinking about coherence may also be relevant to epistemology.

\section{A Variant-specific probabilities}

In the treatment of two cultural variants that influence each other in section 5, I assumed that the probabilities of direct transmission and internal inference were the same for both cultural variants. I dispense with that assumption here. (Due to length constraints, this appendix might not appear in the published version of this paper.)

For two cultural variants $C_{1}$ with frequency $x$ and $C_{2}$ with frequency $y$, let $p$ be $C_{1}$ 's direct transmission probability, and let $q$ be $C_{2}$ 's, and let $r$ and $s$ be $C_{1}$ 's and $C_{2}$ 's respective internal inference probabilities. Assume that all of these probabilities lie within $(0,1)$. Extending equations (7) and (8), the frequencies in the next generation are:

$$
\begin{aligned}
& x^{\prime}=1-(1-p x)(1-r q y)=p x+r q y-r p q x y \\
& y^{\prime}=1-(1-q y)(1-s p x)=q y+s p x-s p q x y .
\end{aligned}
$$

To derive equilibria, we can set $x^{\prime}=x$ and $y^{\prime}=y$, and then solve for $y$ in (13) and for $x$ in (14):

$$
y=\frac{(1-p) x}{r q-r p q x} \quad x=\frac{(1-q) y}{s p-s p q y}
$$

We substitute the value of $y$ from the left-hand equation into the right-hand equation, and simplify to get:

$$
x=\frac{(1-p)(1-q) x}{s p(r q-r p q x)-s p q(1-p) x}=\frac{(1-p)(1-q) x}{r s p q-s p q(r p+(1-p)) x}
$$

This holds when $x=0$, so there is an equilibrium at zero. When $x>0$, we can divide by $x$, and multiply by the denominator to get:

$$
r s p q-\operatorname{spq}(r p+(1-p)) x=(1-p)(1-q) .
$$

There is thus an equilibrium at

$$
\hat{x}=\frac{r s p q-(1-p)(1-q)}{s p q(r p+(1-p))} .
$$


A nonzero equilibrium for $y$ can be derived in the same way:

$$
\hat{y}=\frac{r s p q-(1-p)(1-q)}{r p q(s q+(1-q))} .
$$

These equilibria need not be equal, though it follows from (13) and (14) that when either is zero, they both are. For example, as $y$ approaches zero, (13) approximates $x^{\prime}=p x$, which goes to zero when iterated (cf. \$2). Note that the denominators in (15) and (16) are positive, since $r, s, p, q \in(0,1)$. So there are a nonzero equilibria for both $x$ and $y$ iff $r s p q>(1-p)(1-q)$, or

$$
r s>\left(\frac{1-p}{p}\right)\left(\frac{1-q}{q}\right) .
$$

When $p=q$ and $r=s$, taking the square root of equation (17) shows that it reduces to (12), as expected. To see that (15) reduces to (11), note that the numerator in (15) is equal to $r^{2} p^{2}-p^{2}+2 p-1$ under the same conditions. This quantity is equal to:

$$
(r p+p-1)(r p-p+1) .
$$

Thus when $r=s$ and $p=q$, (15) becomes

$$
\hat{x}=\frac{(r p+p-1)(r p-p+1)}{r p^{2}(r p-p+1)},
$$

which reduces to (11).

\section{References}

Angere, Staffan (2008). Coherence as a heuristic. Mind 117(465):1-26.

URL http://mind .oxfordjournals . org/content/117/465/1. abstract

BonJour, Laurence (1985). The Structure of Empirical Knowledge. Harvard.

Bransford, John and National Research Council, (U.S.) (2000). How People Learn : Brain, Mind, Experience, and School. National Academy Press.

Enquist, Magnus; Strimling, Pontus; Eriksson, Kimmo; Laland, Kevin; and Sjostrand, Jonas (2010). One cultural parent makes no culture. Animal Behaviour 79:1353-1362.

Fitelson, Branden (2003). A probabilistic theory of coherence. Analysis 63:194-199. 
Fitelson, Branden (2004). Two technical corrections to my coherence measure. (From the author's website, fitelson.org. Downloaded March 1, 2014.). URL http://fitelson.org/coherence2.pdf

Godfrey-Smith, Peter (2009). Darwinian Populations and Natural Selection. Oxford University Press, Oxford, UK.

Haigh, John (1978). The accumulation of deleterious genes in a populationMuller's ratchet. Theoretical Population Biology 14:251-267.

Henrich, Joseph; Heine, Steven J.; and Norenzayan, Ara (2010). The weirdest people in the world? Behavioral and Brain Sciences 33:61-83.

Lehrer, Keith (2000). Theory of Knowledge. Westview, 2nd ed.

Lewis, C. I. (1946). An Analysis of Knowledge and Valuation. Open Court Publishing. Maynard Smith, John (1978). The Evolution of Sex. Cambridge University Press.

Olsson, Erik J. (2005). Against Coherence: Truth, Probability, and Justification. Oxford University Press, Oxford, UK.

Otto, Sarah P. and Lenormand, Thomas (2002). Resolving the paradox of sex and recombination. Nat Rev Genet 3(4):252-261.

Pearl, Judea (2009). Cauality: Models, reasoning, and Inference. Cambridge, 2nd ed.

Richerson, Peter J. and Boyd, Robert (2005). Not By Genes Alone. Oxford University Press, Oxford, UK.

Rogers, Alan R. (1988). Does biology constrain culture? American Anthropologist 90(4):819-831.

Schupbach, Jonah N. (2011). New hope for Shogenji's coherence measure. British Journal for the Philosophy of Science 62:125-142.

Shogenji, Tomoji (1999). Is coherence truth conducive? Analysis 59.4:338-345.

Waxman, David and Loewe, Laurence (2010). A stochastic model for a single click of Muller's ratchet. Journal of Theoretical Biology 264:1120-1132.

Wimsatt, William C. (2007). Re-Engineering Philosophy for Limited Beings: Piecewise Approximations to Reality. Harvard University Press. 